\title{
Irinotecan and colorectal cancer: the role of p53, VEGF-C and $\alpha$-B-crystallin expression
}

\author{
Jessica Weekes • Yik-Hong Ho • Sabe Sebesan • \\ Kate Ong • Alfred King-Yin Lam
}

Accepted: 17 November 2009 /Published online: 10 December 2009

(C) Springer-Verlag 2009

\section{Dear Editor:}

We would like to echo and provide additional information on the review paper by Mitry et al. in Int J Colorectal Dis 2009;24:605-621, which examined the literature of irinotecan as palliative therapy for metastatic colorectal cancer. The conclusions of the paper are irinotecan therapy clearly improves outcome of the patients and genetic markers may provide more information about the treatment.

In our practice, irinotecan is the common second line chemotherapy used for failed first line chemotherapy treatment for colorectal cancer. However, irinotecan is not without side effects, and not every patient registers a response to irinotecan. In the literature, there is lack of study to investigate the factors at molecular level, which can predict the responsiveness of the advanced stages or recurrent colorectal cancer to the irinotecan. Thus, we would like to present our pilot data on the use of molecular makers that may help to identify the patients who can be benefited from irinotecan.

The clinicopathological features of 20 patients with advanced or recurrent colorectal adenocarcinoma who were treated by irinotecan were studied. The findings were correlated with the expression of the molecular markersp53, vascular endothelial growth factor (VEGF) and $\alpha$-B-

\author{
J. Weekes $\cdot$ Y.-H. Ho $\cdot$ K. Ong \\ Surgery (School of Medicine) and North Queensland Centre \\ for Cancer Research (Australian Institute of Tropical Medicine), \\ James Cook University, \\ Townsville, QLD 4811, Australia \\ S. Sebesan \\ Medical Oncology, Townsville Hospital, \\ Townsville, QLD 4811, Australia \\ A. K.-Y. Lam $(\bowtie)$ \\ Pathology, Griffith Medical School, Gold Coast Campus, \\ Gold Coast, QLD 4222, Australia \\ e-mail: a.lam@griffith.edu.au
}

crystallin in the colorectal cancer as detected by immunohistochemistry. These markers were chosen because they play roles in tumour apoptosis and angiogenesis.

Of the 20 patients, $5 \%(n=1)$ responded completely, $30 \%(n=6)$ partially responded, $40 \%(n=8)$ had stable disease and $25 \%(n=5)$ had progressive disease. Thus, the patients were classified as responders (responded completely or partially responded) to irinotecan treatment in $35 \%$ of patients overall $(n=7)$. All the patients died of causes related to the colorectal cancer. Response to irinotecan significantly correlated with a longer median survival $(p=$ 0.008 , log-rank test). Median survival for non-responders being 19 months from the commencement of irinotecan till death compared to responders that had a median survival of 39 months. Survival of the patients with advanced stages colorectal cancer was only related to response to irinotecan and not to other clinicopathological factors.

The colorectal cancers were positive for p53, VEGF and $\alpha$ B-crystallin in $80 \%(n=16), 65 \%(n=13)$ and $0 \%$. In tumours positive for $\mathrm{p} 53,38 \%$ were responders to irinotecan, and in tumours negative for p53, 25\% were responders. Also, in tumours positive for VEGF, 39\% were responders to irinotecan, whereas in tumours negative for VEGF, 29\% were responders. Thus, tumours positive for $\mathrm{p} 53$ or VEGF are more likely to be responsive to irinotecan. It supports the notion by the paper that genetic markers may provide information to predict treatment outcome with irinotecan. Nevertheless, the number of cases in this pilot study was small, and the statistical analysis failed to show unequivocal significant value.

Immunohistochemical approach at tissue level is a simple and economical way to test the roles of genetic markers in predicting cancer response to chemotherapy. Thus, in the future, a personalised approach may be applied to patients with advanced or recurrent colorectal cancer based on molecular makers. Further studies on a larger scale using markers like p53 and VEGF are needed to confirm the hypothesis. 